\title{
Prevalencia y Factores de riesgo de ansiedad en estudiantes de Medicina de la Fundación Universitaria Juan N. Corpas. Un estudio de corte transversal.
}

\author{
Lina M. Pinilla ${ }^{1 *}$, Oscar J. López², Daniela A. Moreno ${ }^{3}$, Jersson C. Sánchez ${ }^{4}$.
}

\begin{abstract}
${ }^{1}$ Docente titular Fundación Universitaria Juan N. Corpas. Grupo de Investigación en Terapéuticas Alternativas y Farmacología Vegetal. Bogotá. Colombia ${ }^{2}$ Docente titular Fundación Universitaria Juan N. Corpas. Grupo de Investigación en Terapéuticas Alternativas y Farmacología Vegetal. Bogotá. Colombia. ${ }^{3}$ Estudiante de VI año de Medicina, Facultad de Medicina, Fundación Universitaria Juan N Corpas. Semillero de Investigación en Terapéuticas Alternativas y Farmacología Vegetal. Bogotá. Colombia.

${ }^{4}$ Estudiante de VI año de Medicina, Facultad de Medicina, Fundación Universitaria Juan N Corpas. Semillero de Investigación en Terapéuticas Alternativas y Farmacología Vegetal. Bogotá. Colombia.
\end{abstract}

\section{RESUMEN}

Introducción. La ansiedad es el estado de preocupación excesiva, que se produce durante más días de los que ha estado ausente, durante un mínimo de seis meses, en relación con diversos sucesos o actividades (1). En Colombia, la prevalencia en adultos es de 3,9\% (2), en estudiantes universitarios de $9 \%$ (6) a $17 \%$ (7), en estudiantes de ciencias de la salud $26,5 \%$ (8) y en estudiantes de Medicina, del 60\% (9).

La ansiedad es uno de los trastornos que ocasiona mayor discapacidad y años de vida perdidos, compromete el rendimiento académico, favorece la deserción y aumenta el riesgo de consumo de alcohol y sustancias psicoactivas.

Objetivos. Determinar la prevalencia de ansiedad y factores de riesgo asociados, en estudiantes de una facultad de Medicina, con el fin de aportar información para el diseño de estrategias de prevención e intervención.

Metodología. Se realizó un estudio de corte transversal en 1028 estudiantes de Medicina de I a X semestres, matriculados entre julio y diciembre de 2018. Se aplicó un cuestionario estructurado, autoadministrado, que contenía variables sociales, demográficas y académicas, y las correspondientes a la Escala de Ansiedad de Zung y la Escala de Cage para abuso de alcohol. Se determinó la prevalencia de ansiedad global y por semestre y se determinó el Odds Ratio (OR) para cada una de las variables analizadas.

Resultados. $27,52 \%$ de los estudiantes no presentaba ansiedad; $67,80 \%$ ansiedad leve; $3,40 \%$ ansiedad moderada y $1,26 \%$ ansiedad grave

Los factores de riesgo fueron sexo femenino (OR: 2,3353, $p=0.0006$ ), vivir solo (OR: 2,5265, $p=0,0053$ ), menos de 5 horas semanales de esparcimiento (OR: $2,7960, p=0.0008$ ), falta de tiempo para tomar alguna de las comidas (OR: 3,2520, $p=0.0101$ ), dormir menos de 5 horas diarias $(2,1700, p=0,0393)$, sueño no reparador (OR: 3,3949, $p=0,0002$ ), sentirse discriminado (OR: 1,9161, $p=0.0005$ ), desplazamiento hacia la universidad mayor de 90 minutos (OR: 2,2818, $p=0,0025$ ).

Conclusiones. Se encuentra una elevada prevalencia de ansiedad en la población en estudio y se identifican factores de riesgo que pueden aportar información para el diseño de estrategias de prevención y seguimiento.

Palabras clave: Ansiedad, Estudiantes de Medicina, Prevalencia, Factores de riesgo.

\section{ABSTRACT}

Prevalence and Risk Factors of Anxiety in Medical Students of Juan N. Corpas University Foundation. A cross-sectional study.

Introduction. Anxiety is the state of excessive worry, which occurs for more days than it has been absent for a minimum of six months, in relation

to various events or activities (1). In Colombia, the prevalence in adults is $3.9 \%$ (2), in university students from $9 \%$ (6) to $17 \%$ (7), in students of health sciences $26.5 \%$ (8) and in students of Medicine, $60 \%$ (9).

Anxiety is one of the disorders that causes greater disability and years of life lost, compromises academic performance, favors desertion and increases the risk of consumption of alcohol and psychoactive substances.

Objective. To determine the prevalence of anxiety and associated risk factors, in students of a medical school, in order to provide information for the design of prevention and intervention strategies.

Methodology. A cross-sectional study was conducted in 1028 Medicine students from I to X semesters, enrolled between July and December 2018. A structured, self-administered questionnaire was applied, containing social, demographic and academic variables, and those corresponding to the Scale of Zung's Anxiety and the Cage Scale for Alcohol Abuse. The prevalence of global and semester anxiety was determined and the Odds Ratio (OR) was determined for each of the variables analyzed.

Results. $27.52 \%$ of the students did not present anxiety; $67.8 \%$ mild anxiety; $3.4 \%$ moderate anxiety and $1.26 \%$ severe anxiety.

Risk factors were female sex (OR: 2.3353, $\mathrm{p}=0.0006$ ), living alone (OR: 2.5265, p $=0.0053$ ), less than 5 hours per week of leisure (OR: $2.7960, \mathrm{p}=0.0008$ ), lack of time to take any of the meals (OR: 3.2520, $\mathrm{p}$ $=0.0101)$, sleep less than 5 hours a day $(2.1700, p=0.0393)$, unrefreshing sleep (OR: 3.3949, $\mathrm{p}=0.0002)$, feeling discriminated (OR: 1.9161, $\mathrm{p}=$ 0.0005 ), commuting to the university greater than 90 minutes (OR: 2.2818, $\mathrm{p}=0.0025)$.

Conclusions. A high prevalence of anxiety is found in the study population and risk factors are identified that can provide information for the design of prevention and follow-up strategies.

Keywords: Anxiety, Medical Students, Prevalence, Risk factors

$*$ Autor de correspondencia:
Lina M. Pinilla lina.pinilla@juanncorpas.edu.co

Como citar: Pinilla L, López O, Moreno D, Sánchez J. Prevalencia y Factores de riesgo de ansiedad en estudiantes de Medicina de la Fundación Universitaria Juan N. Corpas. Un estudio de corte transversal. Revista Cuarzo 2020;26(1):22-27.

Recibido: 12 de abril de 2020 Aceptado: 2 de junio de 2020 Publicado: 30 de junio de 2020

DOI: https://doi.org/10.26752/cuarzo.v26.n1.493 


\section{INTRODUCCIÓN}

$\mathrm{A}$ nsiedad es el estado de preocupación excesiva, que se produce durante más días de los que ha estado ausente, durante un mínimo de seis meses, en relación con diversos sucesos o actividades (1). Es una condición de agitación desagradable, en la que se tiene la sensación de inquietud y de anticipación de peligros y se presentan síntomas psíquicos y físicos manifestados como reacciones de sobresalto percibidas con claridad por el individuo (2).

Cuando es moderada, la ansiedad proporciona un componente de protección ante situaciones peligrosas, ayuda a mantener la concentración y a enfrentar eficazmente los retos. Pero cuando es desproporcionada o patológica, se manifiesta en ausencia de un peligro reconocido y desencadena alteraciones del funcionamiento físico, psíquico y social, que ocasionan sentimientos de indefensión y de parálisis, con incapacidad para responder (3).

La ansiedad es uno de los problemas de salud mental más frecuentes, se estima que afecta a más de 260 millones de personas en todo el mundo (4).

En Colombia la padece el 3,9\% de la población mayor de 18 años durante toda la vida (5). La prevalencia entre estudiantes universitarios se incrementa a 9\% (6) - 17\% (7), en ciencias de la salud a 26,5\% (8), y en Medicina, hasta el 60\% (9).

Los factores de riesgo en adultos se relacionan con el género femenino, la edad, déficit económico, separación, consumo de alcohol, sustancias psicoactivas, eventos estresantes de la infancia, comorbilidades médicas, antecedentes familiares de enfermedad mental, conflictos en la infancia con los cuidadores y vivir en área rural (10).

Así mismo, las personas que durante la niñez, adolescencia o primeros años de la vida adulta presentan trastornos de ansiedad tienen un mayor riesgo de desarrollar un Trastorno Depresivo Posterior (11).

En estudiantes universitarios se reportan como factores de riesgo aspectos afectivos, económicos, ubicación de la residencia durante el pregrado, vivir lejos de la familia, necesidad de trabajar de manera simultánea a la actividad académica, obligaciones familiares y debilidad de las redes de apoyo social (12).

Particularmente en las áreas de Ciencias de la Salud, son factores predisponentes las prácticas clínicas, la realización de turnos, los cambios en los ciclos de sueño, dificultades económicas, desempeñar actividades laborales, tener obligaciones familiares, debilidad de redes de apoyo social y deterioro de la salud mental (13)

Considerando la alta prevalencia de ansiedad entre la población general y los resultados de estudios que sugieren una frecuencia aún mayor entre los estudiantes universitarios de ciencias de la salud, se realizó una investigación con el objetivo de determinar la prevalencia de ansiedad y los principales factores de riesgo asociados, en un grupo estudiantes de Medicina, con el fin de aportar información que oriente el diseño de estrategias de prevención e intervención. Los objetivos específicos fueron: Identificar las características sociales y demográficas de la población en estudio, Establecer la prevalencia de ansiedad global, Establecer la prevalencia de ansiedad por semestre y Determinar los principales factores asociados a ansiedad.

De acuerdo con los estudios disponibles, se esperaba encontrar una alta prevalencia de ansiedad en la población en estudio e identificar algunos factores de riesgo asociados.

\section{MATERIALES Y MÉTODOS}

Se realizó un estudio de corte transversal en una Facultad de Medicina de carácter privado, localizada en la ciudad de Bogotá, Colombia.

Se convocó a participar en la investigación a la totalidad de los estudiantes activos matriculados entre I y X semestres, en el período julio - diciembre de 2018. Mediante muestreo no probabilístico fueron seleccionados los que se encontraran desarrollando sus actividades académicas en el campus de la Universidad. Se solicitó a quienes aceptaron voluntariamente participar, la firma del consentimiento informado.

Se realizó la aplicación de un cuestionario estructurado y auto administrado, que contenía las variables en estudio, la Escala de Ansiedad de Zung y la Escala de Cage para abuso de alcohol (Anexo 1)

Las variables independientes fueron: Reprobación de asignatura, Control de nota, Crédito estudiantil, Trabajo, Disgusto por asignaturas y Sentimiento de discriminación, medidas a nivel nominal dicotómico. Lugar de residencia, personas con las que convive, actividades en tiempo libre, Comidas que consume diariamente, Motivos de discriminación, medidas a nivel nominal politómico. Tiempo de estudio en casa, Tiempo de esparcimiento, Tiempo de sueño, Calidad del sueño, Actividad física, Tiempo de desplazamiento a la Universidad, medidas a nivel ordinal. Edad y Turnos por mes, medidas a nivel de razón.

La variable de desenlace fue Ansiedad, medida a nivel ordinal: Sin Ansiedad, Ansiedad leve, Ansiedad moderada y Ansiedad grave, de acuerdo con la escala de Zung.

La encuesta fue respondida de manera anónima, en un tiempo aproximado de 15 minutos. Los datos se tabularon en una hoja de Excel y posteriormente fueron exportados para análisis al programa SPSS.

Se realizó la estadística descriptiva y se estimó la prevalencia de ansiedad global y por semestre. Como medida de asociación 
se estimó el Odds Ratio, considerando una significancia estadística del 95\% ( $p<0.05)$.

Aspectos éticos: Según la Resolución 8430 de 1993 la investigación se clasifica como Sin riesgo.

Dada la participación de estudiantes, se solicitó la firma del consentimiento informado (Anexo 2). El protocolo fue aprobado por el Comité de Ética de la Institución.

\section{RESULTADOS}

En la encuesta participaron 1049 estudiantes $(64,79 \%)$ de los 1619 matriculados entre I y X semestres en el período julio diciembre de 2018.

Con el fin de reducir sesgos de información fueron excluidos 21 cuestionarios en los que se identificó incoherencia en los datos suministrados por los estudiantes, respuestas duplicadas y registro incompleto o puntajes iguales en las variables de la escala de Zung. El análisis final se efectuó sobre 1028 estudiantes, distribuidos entre primer semestre $(n=134)$, segundo $(n=86)$, tercero $(n=108)$, cuarto $(n=127)$, quinto $(n=77)$, sexto $(n=63)$, séptimo $(n=123)$, octavo $(n=100)$, noveno $(n=105)$ y décimo $(n=105)$.

El $68,9 \%$ de la población corresponde a mujeres, la edad mínima es de 16 años, la máxima de 35 (promedio 20,5, DE 2,426), 98,2\% son solteros.

El 41,6\% cuenta con crédito estudiantil, 8,5\% trabaja además de estudiar.

El 31,6\% cambió de lugar de residencia al comenzar sus estudios universitarios. Entre estos, el $25,6 \%$ procedía de Cundinamarca, Tolima, Meta o Boyacá. 7 estudiantes $(0,94 \%)$ eran procedentes de otros países.

$14,4 \%$ de los estudiantes viven solos, en una casa o en una residencia estudiantil, el $4,8 \%$ con amigos y el $80,8 \%$ con familiares (madre 63,1\%, padre 52,8\%, hermanos $50,3 \%$, abuelos, tíos o primos $19,9 \%$ ).

En casa, el $64 \%$ estudia entre 6 y 15 horas semanales, el 10,2\% más de 22 horas y el 10,1\% menos de 5 horas.

El tiempo dedicado a actividades de esparcimiento es de 6 a 10 horas semanales en el $42,8 \%$, menos de 5 en el 38,5\% y más de 22 en el $1,6 \%$.

En el tiempo libre, las actividades que se realizan con mayor frecuencia son ver televisión o películas en casa 77,6\%, salir con amigos $44,7 \%$, jugar en internet $37,7 \%$, practicar algún deporte $36,8 \%$, leer $35 \%$, desarrollar actividades artísticas $16 \%$, manualidades $9,3 \%$.

El $79 \%$ de los estudiantes desayuna, $86,4 \%$ almuerza y $79,7 \%$ come. Medias nueves toma el $62 \%$ y onces el $43 \%$. Quienes no consumen alguna de las comidas, lo hacen por falta de tiempo en $59,2 \%$ de los casos, falta de apetito en $33,8 \%$, falta de costumbre $10 \%$, razones económicas $17,5 \%$, para evitar el sobrepeso en $11,5 \%$

Durante la carrera, el $58 \%$ ha sentido disgusto por alguna asignatura. En el $57 \%$ por causas relacionadas con el docente, $36,2 \%$ por considerarla de poca importancia para la vida profesional, $27,2 \%$ por dificultad para aprender los temas.

El tiempo de sueño durante el período académico es de 6 a 7 horas diarias en el 28,4\%, 4 a 5 horas en el $62,7 \%$, menos de 3 horas en el 4,6\% y más de 7 horas en el 4,3\%. El 45,6\% considera que el sueño no es reparador, nunca o casi nunca.

$36,9 \%$ de los estudiantes hace ejercicio 2 a 3 veces por semana, $19 \%$ más de 4 veces, 48,6\% no realiza actividad física o lo hace una sola vez por semana.

El tiempo de desplazamiento a la Universidad es menor de media hora en el $30,5 \%$, de media a una hora en el $43,8 \%$, de una a dos horas en el $20,8 \%$ y mayor de dos horas en el $4,9 \%$.

El $22,2 \%$ se ha sentido discriminado o excluido. De este porcentaje, el $28,9 \%$ por su forma de pensar, $25,4 \%$ por apariencia física, 8,3\% por razones económicas, $6,1 \%$ por forma de vestir, 5,3\% por razones religiosas, 3,1\% por orientación sexual.

La percepción de discriminación aumenta en forma gradual desde $6 \%$ de la población en primer semestre a 34,6\% en décimo semestre (Gráfico 1).

\section{Gráfico 1: Percepción de discriminación o} exclusión, por semestre.

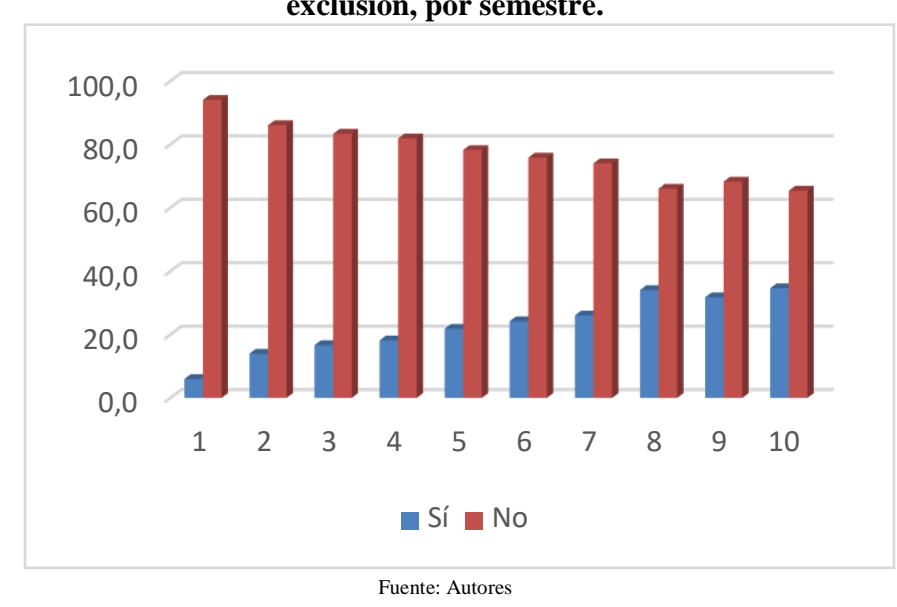

El $43 \%$ de los estudiantes no consume bebidas alcohólicas. El $34,5 \%$ bebe, pero no alcanza el puntaje mínimo para consumo de riesgo en la Escala de CAGE. 11,6\% son bebedores sociales, $6,9 \%$ tienen consumo de riesgo, $3 \%$ consumo perjudicial y $1,0 \%$ dependencia alcohólica.

\section{Prevalencia de ansiedad}

De acuerdo con la escala de Zung, el 27,52\% de los estudiantes no presenta ansiedad, el 67,8\% presenta ansiedad leve, el 3,40\% ansiedad moderada y el 1,26\% ansiedad grave. 
Los semestres en los que se presenta mayor proporción de estudiantes con algún grado de ansiedad son: sexto $(87,1 \%)$, segundo $(80,4 \%)$, cuarto $(78 \%)$ y octavo $(75 \%)$.

Si se consideran solamente los casos de ansiedad moderada y grave, los semestres con mayores proporciones son cuarto $(10,3 \%)$, décimo $(6,7 \%)$, quinto $(6,4 \%)$, noveno $(4,8 \%)$, séptimo $(4,9 \%)$ y segundo $(3,5 \%)$.

La mayor proporción de estudiantes sin ansiedad se encuentra en primer semestre, con $38,1 \%$. (Tabla 1 ).

\section{Factores asociados a ansiedad}

No se encontró asociación entre ansiedad y estado civil, ciclo de formación médica (básico o clínico), tener crédito para financiar los estudios, cambiar de residencia al iniciar los estudios, trabajar, sentir disgusto por alguna asignatura y consumo de bebidas alcohólicas.

Se encontró asociación entre ansiedad y sexo femenino, vivir solo, contar con menos de 5 horas semanales de esparcimiento, falta de tiempo para tomar alguna de las comidas, dormir menos de 5 horas diarias, sueño no reparador, sentirse excluido o discriminado y requerir tiempo mayor de 90 minutos en el desplazamiento hacia la universidad.

El tiempo de estudio en casa mayor de 22 horas semanales se asoció a reducción del riesgo de ansiedad (Tabla 2).

Tabla 2: Odds Ratio de factores asociados a ansiedad.

\begin{tabular}{|c|c|c|c|}
\hline & Odds Ratio & IC 95\% & $\mathrm{p}$ \\
\hline Estado civil casado & 2.6168 & $0.5842-11.7217$ & 0.2086 \\
\hline $\begin{array}{r}\text { Ciclo de formación } \\
\text { clínica }\end{array}$ & 0.8957 & $0.4954-1.6195$ & 0.7155 \\
\hline Cambio de residencia & 1.1947 & $0.6513-2.1915$ & 0.5655 \\
\hline Tener crédito & 1.1992 & $0.6702-2.1459$ & 0.5405 \\
\hline Trabajar & 0.9814 & $0.3441-2.7988$ & 0.9720 \\
\hline $\begin{array}{r}\text { Disgusto por alguna } \\
\text { asignatura }\end{array}$ & 1.6285 & $0.8731-3.0374$ & 0.374 \\
\hline $\begin{array}{r}\text { Actividad física } 1 \mathrm{vez} \\
\text { por semana o no } \\
\text { realiza actividad } \\
\text { física }\end{array}$ & 1.2668 & $0.7082-2.2658$ & 0.4254 \\
\hline Sexo femenino & 1.6551 & $1.2419-2.2057$ & $0.0006^{*}$ \\
\hline Vivir solo & 2.5265 & $1.3171-4.8462$ & $0.0053^{*}$ \\
\hline $\begin{array}{r}\text { Menos de } 5 \text { horas } \\
\text { semanales de } \\
\text { esparcimiento }\end{array}$ & 2.7960 & $1.5367-5.0871$ & $0.0008 *$ \\
\hline $\begin{array}{r}\text { Falta de tiempo para } \\
\text { tomar alguna de las } \\
\text { comidas }\end{array}$ & 3.2520 & $1.3244-7.9852$ & $0.0101^{*}$ \\
\hline $\begin{array}{r}\text { Dormir menos de } 5 \\
\text { horas diarias }\end{array}$ & 2.1700 & $1.0386-4.5339$ & $0.0393^{*}$ \\
\hline $\begin{array}{r}\text { Sueño reparador } \\
\text { nunca o casi nunca }\end{array}$ & 3.3949 & $1.7741-6.4965$ & $0.0002 *$ \\
\hline $\begin{array}{r}\text { Sentirse excluido o } \\
\text { discriminado }\end{array}$ & 1.9161 & $1.3270-2.7668$ & $0.0005^{*}$ \\
\hline $\begin{array}{r}\text { Tiempo de } \\
\text { desplazamiento a la } \\
\text { Universidad mayor } \\
\text { de } 90 \text { minutos }\end{array}$ & 2.2818 & $1.3356-3.8982$ & $0.0025^{*}$ \\
\hline $\begin{array}{r}\text { Estudiar en casa más } \\
\text { de } 22 \text { horas } \\
\text { semanales }\end{array}$ & 0.0153 & $0.0059-0.0395$ & $<0.0001^{*}$ \\
\hline
\end{tabular}

Una alta proporción de estudiantes dispone de menos de 5 horas de esparcimiento a la semana. Se observa tendencia a la reducción en las horas de descanso en semestres superiores. La actividad más frecuente en el tiempo libre es ver televisión o películas en casa. Se observa baja frecuencia de práctica deportiva, lectura y actividades artísticas.

El 46.8\% no realiza actividad física o lo hace menos de una vez por semana. Aunque no se encontró asociación de este factor con ansiedad, se considera importante su intervención como factor de riesgo para otros desenlaces.

En la mayoría de estudiantes el tiempo de sueño es inferior a 5 horas y es considerado no reparador. Estos dos factores tuvieron asociación positiva con ansiedad.

La falta de tiempo para realizar actividades como tomar alimentos es un factor común para un gran número de estudiantes. Debe investigarse si obedece a un exceso de labores académicas o si se trata de una percepción originada en la inadecuada organización de las actividades por parte del estudiante.

Interpretación de los resultados: Aunque el estudio convocó a la totalidad de los estudiantes activos, la ausencia de algunos grupos en el campus de la Universidad durante el período en 
que se aplicaron las encuestas ocasionó limitaciones en la interpretación y en la generalización de los resultados.

En el grupo participante, los hallazgos de prevalencia de ansiedad, son semejantes a los reportados en estudiantes de Medicina, lo cual plantea interrogantes sobre aquellas características inherentes a la carrera, independientemente del contexto, que son susceptibles de producir sobrecarga emocional y no son modificables. La intervención en estos casos estaría orientada a promover en los estudiantes estrategias de afrontamiento, preferiblemente durante los primeros semestres de estudio.

Desde el punto de vista estadístico, se encontró asociación entre los nueve factores descritos y la presencia de ansiedad. Sin embargo, se identifican en la población en estudio otras características que ameritan análisis y posible intervención, tal es el caso del uso del tiempo libre, la actividad física y el consumo de bebidas alcohólicas.

Limitaciones del estudio: A pesar del alto número de participantes, la proporción de estudiantes que no fue incluida en el estudio, bien sea por no encontrarse en el campus de la Universidad o por decisión voluntaria, podría ocasionar un sesgo de información, por lo que los resultados obtenidos son aplicables solamente al grupo en estudio.

Se recomienda en futuros estudios, considerar la realización de entrevistas personales con uso de escalas hetero administradas de mayor especificidad para el diagnóstico de ansiedad en sus diferentes niveles.

Se hace necesaria también una aproximación a motivaciones, actitudes y percepciones mediante investigaciones conenfoque cualitativo.

\section{CONCLUSIONES}

El presente estudio identifica nueve factores asociados a ansiedad, la mayoría susceptibles de ser modificados. Se sugiere evaluar intervenciones dirigidas a ampliar las estrategias de apoyo a estudiantes que viven solos, revisar horarios de actividades en el campus para brindar tiempo suficiente para descansar y comer, fortalecer programas por el respeto y la tolerancia, mejorar la calidad del tiempo libre, brindar educación sobre calidad e higiene del sueño y asesorar sobre la adecuada organización de los tiempos de estudio y descanso.

Se espera que los resultados aporten información para fortalecer estrategias de prevención, en su propósito de procurar el bienestar integral de la comunidad universitaria

Conflicto de Interés: Los investigadores declaran no tener ningún conflicto de interés en la realización del estudio.

Financiación: El estudio fue financiado en su totalidad por la
Fundación Universitaria Juan N. Corpas.

\section{REFERENCIAS}

1. American Psychiatric Association. Guía de consulta de los Criterios Diagnósticos del DSM-5. Washington; 2014

2. Sierra J, Ortega V, Zubeidat I. Ansiedad, angustia y estrés: tres conceptos a diferenciar. Rev. Mal-Estar Subj. 2003; (3):10-59. Disponible en http://pepsic.bvsalud.org/pdf/malestar/v3n1/02.pdf

3. Riveros M, Hernández H, Rivera J. Niveles de Depresión y ansiedad en estudiantes universitarios de Lima Metropolitana. Revista IPSI. 2007; (10):91-102.

Disponible

en https://revistasinvestigacion.unmsm.edu.pe/index.php/psico /article/view/3909/3133

4. Organización Mundial de la Salud. [Internet]. 2017. Acceso 5 de noviembre de 2019. Disponible en https://www.who.int/mental_health/es/

5. Ministerio de Salud de Colombia. Encuesta Nacional de Salud mental, 2015. Tomo I. Disponible en http://www.odc.gov.co/Portals/1/publicaciones/pdf/consum o/estudios/nacionales/CO031102015-

salud_mental_tomoI.pdf

6. Universidad Industrial de Santander (UIS), Departamento de Bienestar Universitario. Reporte de consultas atendidas según trastorno y/o diagnóstico establecido en el año 2004. Documento de trabajo inédito. Departamento de Bienestar Universitario, Universidad Industrial de Santander (UIS), Bucaramanga, 2005.

7. Universidad Pontificia Bolivariana (UPB), Departamento de Bienestar Universitario. Reporte de consultas atendidas según trastorno y/o diagnóstico establecido en los meses de junio a septiembre del año 2005. Documento de trabajo inédito, Departamento de Bienestar Universitario, Universidad Pontificia Bolivariana, Bucaramanga, 2005.

8. Caro Y, Trujillo S, Trujillo, N. Prevalencia y factores asociados a sintomatología depresiva y ansiedad rasgo en estudiantes universitarios del área de la salud. Psychologia. 2019; 13(1), 41-52.

9. Bohórquez A. Prevalencia de depresión y ansiedad según las escalas de Zung y evaluación de la asociación con el desempeño académico en los estudiantes de medicina de la Pontificia Universidad Javeriana. Bogotá: Pontificia Universidad Javeriana. 2007. Disponible en https://www.researchgate.net/publication/49175486_Preval encia_de_depresion_y_de_ansiedad_segun_las_escalas_de _Zung_y_evaluacion_de_la_asociacion_con_el_desempeno _academico_en_los_estudiantes_de_medicina_de_la_Ponti ficia_Universidad_Javeriana/citation/download

10. Gómez C, Tamayo N. Bohórquez A, Rondónd M, Medina M. Trastornos depresivos y de ansiedad y factores asociados en la población adulta colombiana, Encuesta Nacional de Salud Mental; 2015. Revista Colombiana de Psiquiatría, 45(S1), pp.58-67.

11. Grupo de Trabajo de la Guía de Práctica Clínica para el Manejo de Pacientes con Trastornos de Ansiedad en Atención Primaria. Madrid: Plan Nacional para el SNS del MSC. Unidad de Evaluación de Tecnologías Sanitarias. Agencia Laín Entralgo. Comunidad de Madrid; 2008. Guías de Práctica Clínica en el SNS: UETS Nº 2006/10

12. Agudelo D, Casadiegos C, Sánchez, D. Características de ansiedad y depresión en estudiantes universitarios. International Journal of Psychological Research, 2008; 1(1): 34-39 
13. Brewer T. Test-taking anxiety among nursing and general collage students. Journal of Psychosocial Nursing and Mental Health. 2002; 40 (11): 22-31.

14. Osada J, Rojas M, Rosales C, Vega-Dienstmaier J. Sintomatología ansiosa y depresiva en estudiantes de medicina. Revista de Neuro-Psiquiatría. 2010; 73(1):15-16.

15. Cardona JA., Pérez D, Rivera S, Gómez J, Reyes Á. Prevalence of anxiety in universitary students. Diversitas: Perspectivas en Psicología. 2015; 11(1):79-89.

16. Ogi, C. Perales, A. Uso/Abuso del Alcohol en Estudiantes de Medicina. Anales de la Facultad de Medicina. 2001;62(1): 13-19 
Anexo 1: Escala de Zung para tamización de ansiedad.

\begin{tabular}{|c|c|c|c|c|}
\hline $\begin{array}{c}\text { Marque con una X la } \\
\text { frecuencia con la que } \\
\text { usted presenta las } \\
\text { siguientes } \\
\text { manifestaciones }\end{array}$ & $\begin{array}{l}\text { Nunca o } \\
\text { casi } \\
\text { nunca }\end{array}$ & A veces & $\begin{array}{c}\text { Con } \\
\text { bastante } \\
\text { frecuencia }\end{array}$ & $\begin{array}{l}\text { Siempre } \\
\text { o casi } \\
\text { siempre }\end{array}$ \\
\hline $\begin{array}{l}\text { 1. Me siento más nervioso } \\
\text { y ansioso de lo normal }\end{array}$ & & & & \\
\hline $\begin{array}{r}\text { 2. Me siento con temor sin } \\
\text { razón }\end{array}$ & & & & \\
\hline $\begin{array}{r}\text { 3. Me enojo con facilidad } \\
\text { o siento momentos de mal } \\
\text { humor }\end{array}$ & & & & \\
\hline $\begin{array}{l}\text { 4. Siento como si fuera a } \\
\text { reventar y partirme en } \\
\text { pedazos }\end{array}$ & & & & \\
\hline $\begin{array}{r}\text { 5. Siento que todo está } \\
\text { bien y que nada malo } \\
\text { puede suceder }\end{array}$ & & & & \\
\hline $\begin{array}{r}\text { 6. Me tiemblan las manos } \\
\text { y las piernas }\end{array}$ & & & & \\
\hline $\begin{array}{r}\text { 7. Me molestan los } \\
\text { dolores de la cabeza, } \\
\text { cuello o cintura }\end{array}$ & & & & \\
\hline $\begin{array}{r}\text { 8. Me siento débil yme } \\
\text { canso fácilmente }\end{array}$ & & & & \\
\hline $\begin{array}{l}\text { 9. Me siento tranquilo(a) } \\
\text { y puedo permanecer en } \\
\text { calma fácilmente }\end{array}$ & & & & \\
\hline $\begin{array}{r}\text { 10. Siento que mi corazón } \\
\text { late muy rápido }\end{array}$ & & & & \\
\hline 11. Sufro de mareos & & & & \\
\hline $\begin{array}{r}\text { 12. Sufro de desmayoso } \\
\text { siento que me voya } \\
\text { desmayar }\end{array}$ & & & & \\
\hline $\begin{array}{r}\text { 13. Puedo inspirar y } \\
\text { expirar fácilmente }\end{array}$ & & & & \\
\hline $\begin{array}{r}\text { 14. Siento } \\
\text { adormecimiento y } \\
\text { hormigueo de los dedos } \\
\text { de las manos y de los pies }\end{array}$ & & & & \\
\hline $\begin{array}{l}\text { 15. Sufro de molestias } \\
\text { estomacales o indigestión }\end{array}$ & & & & \\
\hline $\begin{array}{r}\text { 16. Orino con mucha } \\
\text { frecuencia }\end{array}$ & & & & \\
\hline $\begin{array}{r}\text { 17. Generalmente mis } \\
\text { manos están sacas y } \\
\text { calientes }\end{array}$ & & & & \\
\hline $\begin{array}{l}\text { 18. Siento que mi cara } \\
\text { enrojece y me ruborizo }\end{array}$ & & & & \\
\hline $\begin{array}{l}\text { 19. Puedo dormir con } \\
\text { facilidad y descansar } \\
\text { durante la noche }\end{array}$ & & & & \\
\hline 20. Tengo pesadillas & & & & \\
\hline
\end{tabular}

A continuación, encontrará algunas preguntas relacionadas con el consumo de bebidas alcohólicas. Si usted no las consume, por favor deje las casillas en blanco. Si las emplea, responda SI o NO según corresponda.

Si

1. ¿Ha sentido alguna vez que debe beber menos?

2. ¿Le ha molestado que la gente lo critique por su forma de beber? 3. ¿Alguna vez se ha sentido mal o culpable por su forma de beber? 4. ¿Alguna vez ha necesitado beber por la mañana para calmar los nervios o eliminar las molestias por haber bebido la noche anterior?

\section{Anexo 1.2: Cuestionario Básico.}

$$
\text { Pinilla L et al. }
$$

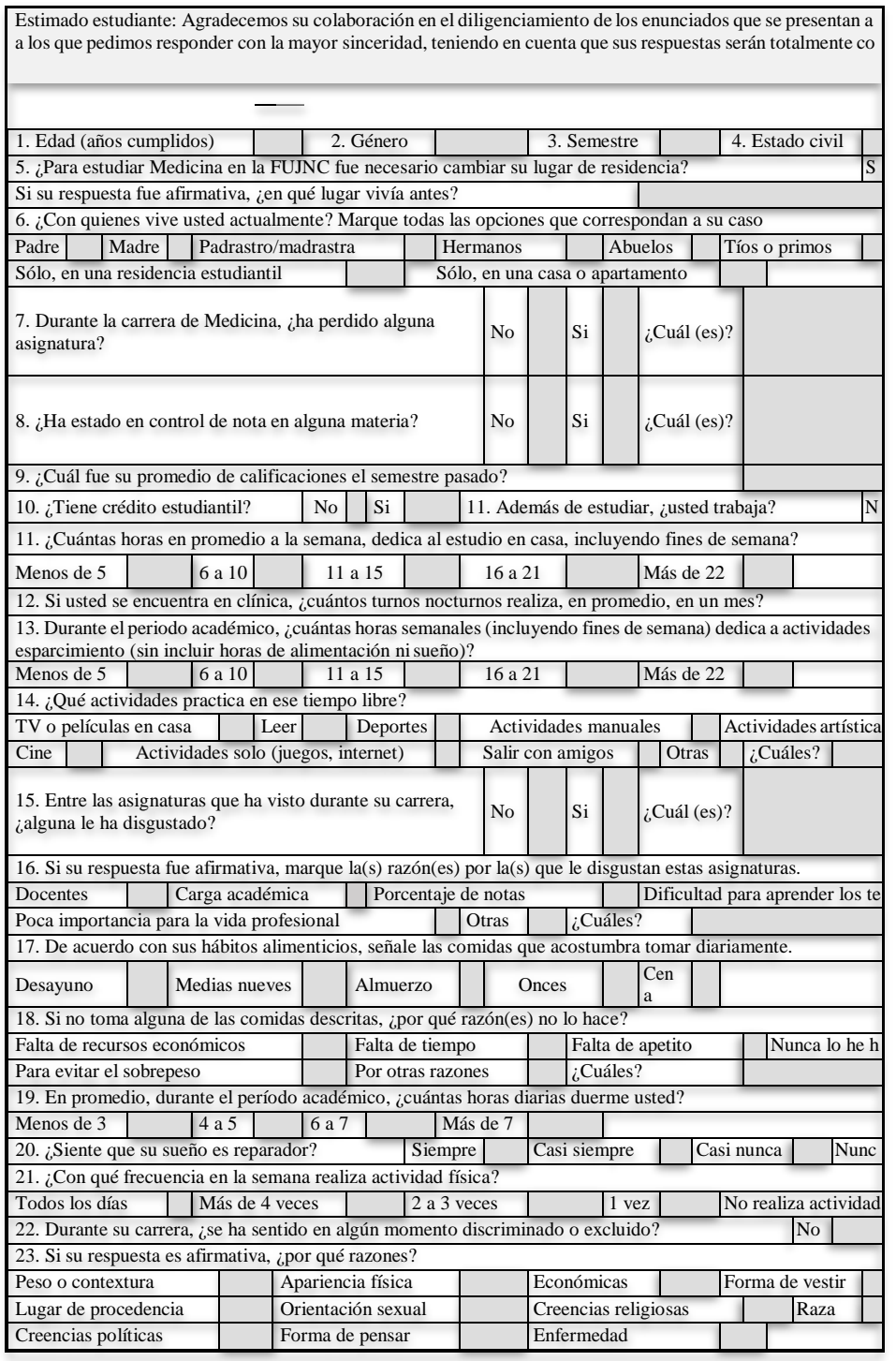

Anexo 2: Consentimiento informado.

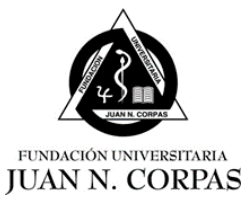

Fundación Universitaria Juan N. Corpas. Facultad de Medicina Semillero de Investigación en Terapéuticas Alternativas.

Consentimiento informado para participación en Investigación.

Apreciado estudiante:

El Semillero de Investigación en Terapéuticas Alternativas desarrolla en la actualidad un proyecto que pretende identificar los factores de riesgo asociados a la presencia de ansiedad en estudiantes de Medicina, y su relación con el desempeño académico. 
Se espera que la información obtenida contribuya al diseño de intervenciones que permitan prevenir, identificar y hacer seguimiento oportuno a los casos y a mejorar el rendimiento académico de los estudiantes.

En este sentido, amablemente le solicitamos diligenciar la siguiente encuesta, que no tiene respuestas correctas, incorrectas, adecuadas o inadecuadas. Solo pretende explorar en qué grado cada una de las frases allí propuestas se identifican con usted.

La información aportada no tendrá efecto en sus calificaciones, no será conocida por estamento universitario alguno diferente al grupo investigador y es absolutamente confidencial. Sus hallazgos solo serán usados para fines académicos e investigativos.

En cumplimiento de los estándares de buenas prácticas en el manejo de datos, su nombre e identificación se volverán anónimos al interior de la base de datos, de tal suerte que en el procesamiento y en los resultados, sus respuestas no permitirán que se le identifique a futuro de ninguna manera.

Si los resultados del estudio son publicados, estos contendrán únicamente información global del conjunto de alumnos participantes.

Acepto participar diligenciando esta encuesta:

SI NO

Nombre:

Identificación: 